\title{
USING MULTIFLOW FORMULATIONS TO SOLVE THE STEINER TREE PROBLEM IN GRAPHS
}

\author{
Laura Bahiense ${ }^{1}$, Arthur Besso $^{2},{\text { Rogerio } \text { Tostas }^{1} \text { and Nelson Maculan }}^{1, *}$
}

\begin{abstract}
We present three different mixed integer linear models with a polynomial number of variables and constraints for the Steiner tree problem in graphs. The linear relaxations of these models are compared to show that a good (strong) linear relaxation can be a good approximation for the problem. We present computational results for the STP OR-Library (J.E. Beasley) instances of type $b, c, d$ and $e$.
\end{abstract}

Mathematics Subject Classification. 90C27, 90Cxx, 90C90.

Received July 20, 2019. Accepted February 21, 2020.

\section{INTRODUCTION}

Using multiflow formulations, we present three mixed integer linear models with a polynomial number of variables and constraints for the Steiner tree problem in graphs. This approach has been used since the 80 's by $[1-6,9,10,13]$.

We study the different models and we compare their linear relaxations to show that a good (strong) linear relaxation can be a good approximation for the problem.

Multiflow formulations with strong linear relaxations are very promising as they can be solved efficiently by good Lagrangian algorithms despite having a large (although polynomial) number of variables and constraints.

We present computational results for the STP OR-Library (J.E. Beasley) instances of type $b, c, d$ and $e$, that can be found at http://people.brunel.ac.uk/ mastjjb/jeb/orlib/steininfo.html.

\section{The Steiner PROBlem In GRAPhS}

The Steiner Problem derives from the Fermat Problem, which consists in, given a triangle, finding the point whose sum of the distances to the vertices is minimal. This problem was generalized to any figure in any dimension is known as the Euclidean Steiner Problem.

The Steiner Problem in Graphs appeared later, and consists in, given a graph $G=(V, E)$, where $V$ is the set of vertices and $E$ is the set of edges (each edge is associated with a cost), and a subset $V_{0} \subseteq V$ of vertices of $G$, finding a connected subgraph $G^{\prime}=\left(V^{\prime}, E^{\prime}\right)$ of minimal cost that contains these vertices.

Keywords. Steiner problem in graphs, multiflow formulations, linear relaxations.

1 Systems Engineering and Computer Science Program, Alberto Luiz Coimbra Institute - Graduate School and Research in Engineering, Federal University of Rio de Janeiro, Rio de Janeiro, Brazil.

2 IBGE Foundation - CRM/GEFET, Rio de Janeiro, Brazil.

*Corresponding author: maculan@cos.ufrj.br 
The vertices of $V_{0}$ are mandatory and are called "terminal points", and the vertices of $V \backslash V_{0}$ are optional. The elements of $V^{\prime} \backslash V_{0}$, i.e., the optional vertices that compose the solution, are known as "Steiner's points". The graph-solution is called the Steiner's Tree, since the existence of cycles always worsens the value of the objective function.

The Steiner problem in graphs is a classical $\mathcal{N} \mathcal{P}$-hard problem $[7,8]$ with many applications in the network design of communication, distribution and transportation systems in general and VLSI design in particular.

\section{MODELS}

Let $G=(V, E)$ be a connected graph, where $V=\{1,2,3, \ldots, n\}$ is the set of vertices or nodes, and $E$ is the set of edges. Let $G_{d}=(V, A)$ be a directed graph derived from $G$, where $A=\{(i, j),(j, i) \mid\{i, j\} \in E\}$, and each edge $u=\{i, j\} \in E$ is associated with two $\operatorname{arcs}(i, j)$ and $(j, i) \in A$.

We consider $y=\left(y_{u}\right)_{u \in E} \in\{0,1\}^{|E|}, \Gamma^{+}(i)=\{j \mid(i, j) \in A\}, \Gamma^{-}(i)=\{j \mid(j, i) \in A\}, m=|E|, n=|V|$. We also consider $E(i)$ the set of edges $u \in E$ such that an endpoint is $i \in V$. Associated with each edge we have a weight $w_{u}>0, u \in E$.

Let $\left(V_{0}, V_{1}\right)$ be a partition of $V$, that is $V=V_{0} \cup V_{1}, V_{0} \cap V_{1}=\phi$. Supposing $3 \leq\left|V_{0}\right|<n$, we want to find a connected sub-graph of $G, G_{T}=\left(V_{T}, E_{T}\right)$, where $V_{0} \subset V_{T}$ such that $\sum_{u \in E_{T}} w_{u} y_{u}$ is minimum. It is easy to see that $G_{T}$ is a sub-tree of $G$, defined as a Steiner tree.

A vertex $s \in V_{0}$ is chosen to be the source offering $\left|V_{0} \backslash\{s\}\right|$ commodities for the remaining terminal vertices. Variables $z_{i j}^{k} \geq 0,(i, j) \in A, k \in V_{0} \backslash\{s\}$ indicate the continuous flow amount of commodity $k$ going through arc $(i, j)$ having $s$ as source and $k$ as terminal.

In this paper we are looking for a special connected subgraph of $G$, that contains all points of $S$ with minimum weight. This subgraph will be a minimum weight tree since $w_{u}>0, u \in E[11]$.

\subsection{First model $-\mathrm{STP}_{1}$}

The first model $\left(\mathrm{STP}_{1}\right)$ is a non-oriented formulation that can be found in [11].

$$
\begin{gathered}
\left(\mathrm{STP}_{1}\right): \min \sum_{\{i, j\} \in E} w_{i j} y_{i j}, \\
\text { subject to : } \\
\sum_{j \in \Gamma^{+}(s)} z_{s j}^{k}=1, \quad k \in V_{0} \backslash\{s\}, \\
\sum_{j \in \Gamma^{+}(k)} z_{k j}^{k}-\sum_{j \in \Gamma^{-}(k)} z_{j k}^{k}=-1, \quad k \in V_{0} \backslash\{s\}, \\
\sum_{j \in \Gamma^{+}(i)} z_{i j}^{k}-\sum_{j \in \Gamma^{-}(i)} z_{j i}^{k}=0, \quad i \in V \backslash\{s, k\}, \quad k \in V \backslash\{s\}, \\
z_{i j}^{k} \leq y_{i j} \text { and } z_{j i}^{k} \leq y_{i j}, \quad\{i, j\} \in E, \quad k \in V_{0} \backslash\{s\}, \\
z_{i j}^{k} \geq 0, \quad(i, j) \in A, \quad k \in V_{0} \backslash\{s\}, \\
y_{i j} \in\{0,1\}, \quad\{i, j\} \in E .
\end{gathered}
$$

Objective function (3.1) minimizes the Steiner tree weight; equations (3.2)-(3.4) represent flow conservation for the terminal vertex $s$ chosen to be the source, for the remaining terminal vertices and for the non-terminal vertices, respectively; constraints (3.5) allow a non-zero flow $z_{i j}^{k}$ or $z_{j i}^{k}$ through an arc $(i, j) \in A$ only if edge $\{i, j\} \in E$ is included in the solution; and, finally, constraints (3.6) and (3.7) define flow variables $z_{i j}^{k}$ as continuous and arc variables $y_{i j}$ as binary, respectively. 


\subsection{Second model $-\mathrm{STP}_{2}$}

If in $\mathrm{STP}_{1}$ we replace constraints (3.5) by

$$
z_{i j}^{k}+z_{j i}^{k} \leq y_{i j}, \quad\{i, j\} \in E, \quad k \in V_{0} \backslash\{s\},
$$

we will have a new model $\mathrm{STP}_{2}$.

\subsection{Third model $-\mathrm{STP}_{3}$}

Model $\left(\mathrm{STP}_{3}\right)$ represents the relationship between flows and arcs in an oriented way: in this formulation, each oriented arc has associated an utilization cost that is independent from the opposite arc, even the value of this cost being equal for both $\operatorname{arcs}\left(w_{i j}=w_{j i}>0,(i, j) \in A\right)$.

In order to distinguish the oriented variables related to the $\operatorname{arcs}$ in $\left(\mathrm{STP}_{3}\right)$, we will change $y_{i j} \in\{0,1\},\{i, j\} \in E$ by $x_{i j} \in\{0,1\},(i, j) \in A$ in this oriented formulation.

$$
\left(\mathrm{STP}_{3}\right): \min \sum_{(i, j) \in A} w_{i j} x_{i j}
$$

subject to:

Flow constraints (3.2), (3.3), (3.4).

$$
\begin{aligned}
z_{i j}^{k} \leq x_{i j}, & (i, j) \in A, \quad k \in V_{0} \backslash\{s\}, \\
z_{i j}^{k} \geq 0, & (i, j) \in A, \quad k \in V_{0} \backslash\{s\}, \\
x_{i j} \in\{0,1\}, & (i, j) \in A .
\end{aligned}
$$

We note that $(i, j) \in A$ if and only if $(j, i) \in A$. Let $x_{i j}^{*}$ for $(i, j) \in A$ be an optimal solution for $\mathrm{STP}_{3}$. It is easy to observe that $x_{i j}^{*}+x_{j i}^{*} \leq 1$, which means that or $x_{i j}^{*}=x_{j i}^{*}=0$, or $x_{i j}^{*}=0$ and $x_{j i}^{*}=1$, or $x_{i j}^{*}=0$ and $x_{j i}^{*}=1$, since $w_{i j}=w_{j i}>0$. We obtain a minimum directed tree, rooted in $s$, which also contains all points of $S$. This means that we have only one directed path from $s$ to each vertex different from $s$ in the subtree of the original graph $G$ induced by $s$. Therefore, if we find an optimal solution for $\mathrm{STP}_{3}$ we are also solving the indirect Steiner tree problem.

\section{LINEAR RELAXATIONS}

If in $\left(\mathrm{STP}_{1}\right)$ and $\left(\mathrm{STP}_{2}\right)$ we replace $y_{i j} \in\{0,1\},\{i, j\} \in E$, by $0 \leq y_{i j} \leq 1$, and in $\left(\mathrm{STP}_{3}\right)$ we replace $x_{i j} \in\{0,1\},(i, j) \in A$, by $0 \leq x_{i j} \leq 1$, we will have three linear programming relaxations defined by $\left(\operatorname{LSTP}_{1}\right),\left(\operatorname{LSTP}_{2}\right)$ and $\left(\operatorname{LSTP}_{3}\right)$. We call $\operatorname{val}(\cdot)$ the optimum value of the objective function associated with the optimization problem $(\cdot)$.

We know that $\operatorname{val}\left(\mathrm{STP}_{1}\right)=\operatorname{val}\left(\mathrm{STP}_{2}\right)=\operatorname{val}\left(\mathrm{STP}_{3}\right)$, and also $\operatorname{val}\left(\operatorname{LSTP}_{q}\right) \leq \operatorname{val}\left(\mathrm{STP}_{q}\right), q=1,2,3$. We call $\operatorname{val}\left(\operatorname{LSTP}_{q}\right)$ a lower bound of $\operatorname{val}\left(\mathrm{STP}_{q}\right)$. In the next two lemmas we address the strengh of these lower bounds.

Lemma 4.1. $\operatorname{val}\left(\operatorname{LSTP}_{1}\right) \leq \operatorname{val}\left(\operatorname{LSTP}_{2}\right)$.

Proof. In $\left(\operatorname{LSTP}_{1}\right)$ we have:

$$
z_{i j}^{k} \leq y_{i j} \text { and } z_{j i}^{k} \leq y_{i j},\{i, j\} \in E, k \in V_{0} \backslash\{s\}
$$

In $\left(\mathrm{LSTP}_{2}\right)$ we consider:

$$
z_{i j}^{k}+z_{j i}^{k} \leq y_{i j},\{i, j\} \in E, k \in V_{0} \backslash\{s\} .
$$

All feasible solutions of (4.2) are feasible solutions of (4.1), but some solutions of (4.1) are not feasible solutions of (4.2). It means that the set of feasible solutions of $\left(\mathrm{LSTP}_{2}\right)$ is included in the set of feasible solutions of $\left(\mathrm{LSTP}_{1}\right)$, which implies that $\operatorname{val}\left(\mathrm{LSTP}_{1}\right) \leq \operatorname{val}\left(\mathrm{LSTP}_{2}\right)$. 
Lemma 4.2. $\operatorname{val}\left(\mathrm{LSTP}_{2}\right) \leq \operatorname{val}\left(\mathrm{LSTP}_{3}\right)$.

Proof. We know that $w_{i j}=w_{j i}>0$, for all $\{i, j\}$ and $\{j, i\} \in A$. We will prove that at the optimal solutions of $\left(\mathrm{LSTP}_{2}\right)$ and $\left(\mathrm{LSTP}_{3}\right)$ we have:

(i) $z_{i j}^{k} \geq 0$ and $z_{j i}^{k}=0$, or $z_{i j}^{k}=0$ and $z_{j i}^{k} \geq 0$;

(ii) $z_{i j}^{k}+z_{j i}^{k} \leq 1$; and

(iii) $\operatorname{val}\left(\mathrm{LSTP}_{2}\right) \leq \operatorname{val}\left(\mathrm{LSTP}_{3}\right)$.

In order to prove (i), consider by absurd an optimal solution with $z_{i j}^{k}>0$ and $z_{j i}^{k}>0$, which is associated with a circular flow using both $\operatorname{arcs}(i, j)$ and $(j, i) \in A$. Define $d=z_{i j}^{k}-z_{j i}^{k},|d|<1,0 \leq z_{i j}^{k} \leq 1,(i, j) \in A$. Now replace $\bar{z}_{i j}^{k}=d$ and $\bar{z}_{j i}^{k}=0$, if $d \geq 0$; and $\bar{z}_{j i}^{k}=-d$ and $\bar{z}_{i j}^{k}=0$, if $d<0$. These new $\bar{z}_{j i}^{k} \in \mathbb{Z}$ and $\bar{z}_{i j}^{k}+\bar{z}_{j i}^{k} \leq 1$, even for $\left(\mathrm{LSTP}_{3}\right)$. From model $\left(\mathrm{STP}_{2}\right)$ we have the correspondence $\bar{y}_{i j} \geq \bar{z}_{i j}^{k}+\bar{z}_{j i}^{k}$, and from model $\left(\mathrm{STP}_{3}\right)$ the correspondences $\bar{x}_{i j} \geq \bar{z}_{i j}^{k}$ and $\bar{x}_{j i} \geq \bar{z}_{j i}^{k}$. In addition, we also have that $z_{i j}^{k}+z_{j i}^{k} \geq \bar{z}_{i j}^{k}+\bar{z}_{j i}^{k},\{i, j\} \in E$ and $z_{i j}^{k} \geq \bar{z}_{i j}^{k},(i, j) \in A$. Therefore, since $w_{i j}>0$, we have that $w_{i j} y_{i j} \geq w_{i j} \bar{y}_{i j}$, and $w_{i j}\left(x_{i j}+x_{j i}\right) \geq w_{i j}\left(\bar{x}_{i j}+\bar{x}_{j i}\right)$, which is a contradiction.

The proof of (ii) is straightforward: directly from (i) we have that $z_{i j}^{k}+z_{j i}^{k} \leq y_{i j} \leq 1, \forall\{i, j\} \in E$ for $\left(\operatorname{LSTP}_{2}\right)$; and $z_{i j}^{k} \leq x_{i j} \leq 1, \forall(i, j) \in A$ for $\left(\operatorname{LSTP}_{3}\right)$.

Finally, in order to prove (iii), if we define $z_{i j}^{a}=\max _{k \in V_{0} \backslash\{s\}}\left\{z_{i j}^{k}\right\}$ and $z_{j i}^{b}=\max _{k \in V_{0} \backslash\{s\}}\left\{z_{j i}^{k}\right\}$, then:

$$
\left\{\begin{array}{l}
\forall\{i, j\} \in E \text { in }\left(\operatorname{LSTP}_{2}\right): y_{i j}=\max \left\{z_{i j}^{a}, z_{j i}^{b}\right\} ; \text { and } \\
\forall(i, j) \in A \text { in }\left(\operatorname{LSTP}_{3}\right):\left\{\begin{array}{l}
x_{i j}=z_{i j}^{a}, \\
x_{j i}=z_{j i}^{b} .
\end{array}\right.
\end{array}\right.
$$

Therefore, we have that:

$$
\begin{aligned}
& \operatorname{val}\left(\operatorname{LSTP}_{2}\right)=\sum_{\{i, j\} \in E} w_{i j} \max \left\{z_{i j}^{a}, z_{j i}^{b}\right\} ; \text { and } \\
& \operatorname{val}\left(\operatorname{LSTP}_{3}\right)=\sum_{\{i, j\} \in A ; i<j} w_{i j}\left(z_{i j}^{a}+z_{j i}^{b}\right) .
\end{aligned}
$$

Since $z_{i j}^{a}+z_{j i}^{b} \geq \max \left\{z_{i j}^{a}, z_{j i}^{b}\right\}$, we have that $\operatorname{val}\left(\mathrm{LSTP}_{2}\right) \leq \operatorname{val}\left(\mathrm{LSTP}_{3}\right)$.

Corollary 4.3. $\operatorname{val}\left(\mathrm{LSTP}_{1}\right) \leq \operatorname{val}\left(\mathrm{LSTP}_{2}\right) \leq \operatorname{val}\left(\mathrm{LSTP}_{3}\right)$.

Figure 1 shows the strength of $\left(\mathrm{STP}_{3}\right)$ throughout an example of $\operatorname{val}\left(\mathrm{LSTP}_{1}\right)=\operatorname{val}\left(\mathrm{LSTP}_{2}\right)=6.5<$ $\operatorname{val}\left(\mathrm{LSTP}_{3}\right)=\operatorname{val}(\mathrm{STP})=7$ (integer problem value). For $\mathrm{LSTP}_{1}$ and $\mathrm{LSTP}_{2}$ Figure 1a depicts the same fractional solution highlighted in bold: $y_{12}=y_{23}=y_{34}=1, y_{45}=y_{48}=y_{58}=0.5$, the other values of $y_{i j}$ are zero. For $\mathrm{LSTP}_{3}$ Figure 1b shows the integer solution highlighted in bold: $x_{12}=x_{23}=x_{38}=x_{85}=1$, the other values of $x_{i j}$ are zero. The yellow vertices in Figure 1 form the set $V_{0}$, where $s=1$.

\section{Computational Results}

The models presented in Section 3 were tested over the STP OR-Library (J.E. Beasley) for instances of type $b, c, d$ and $e$, that can be found at http://people.brunel.ac.uk/ mastjjb/jeb/orlib/steininfo.html. Table 1 presents the sizes of these instances.

The computing environment consisted of a computer with Intel XeonTM Processor (3.07 GHz, $48 \mathrm{~GB}$ RAM), running under Ubuntu 12.04 LTS operating system. The modeling software used was AMPL version 20150721 (Linux 64 bits) together with the IBM ILOG CPLEX Optimization Studio Interactive Optimizer 12.6.0.0 as solver. 


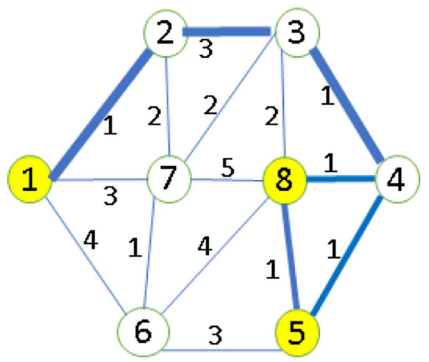

(a)

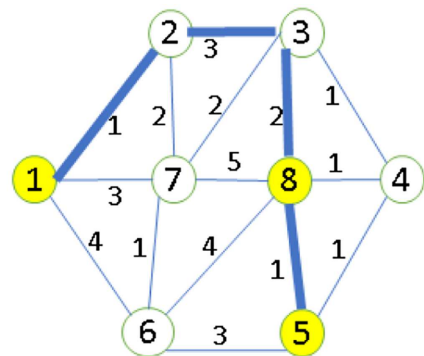

(b)

FIGURE 1. Example of the strength of $\left(\mathrm{STP}_{3}\right): \operatorname{val}\left(\mathrm{LSTP}_{1}\right)=\operatorname{val}\left(\mathrm{LSTP}_{2}\right)<\operatorname{val}\left(\mathrm{LSTP}_{3}\right)$.

TABLE 1. Sizes of instances $b, c, d$ and $e$ from STP OR-Library.

\begin{tabular}{crrrrrrrrrrrrrrrr}
\hline \hline Instance & $|V|$ & $|A|$ & $\left|V_{0}\right|$ & Instance & $|V|$ & $|A|$ & $\left|V_{0}\right|$ & Instance & $|V|$ & $|A|$ & $\left|V_{0}\right|$ & Instance & $|V|$ & $|A|$ & $\left|V_{0}\right|$ \\
\hline$b 1$ & 50 & 63 & 9 & $c 3$ & 500 & 625 & 83 & $d 3$ & 1000 & 1250 & 167 & $e 3$ & 2500 & 3125 & 417 \\
$b 2$ & 50 & 63 & 13 & $c 4$ & 500 & 625 & 125 & $d 4$ & 1000 & 1250 & 250 & $e 4$ & 2500 & 3125 & 625 \\
$b 3$ & 50 & 63 & 25 & $c 5$ & 500 & 625 & 250 & $d 5$ & 1000 & 1250 & 500 & $e 5$ & 2500 & 3125 & 1250 \\
$b 4$ & 50 & 100 & 9 & $c 6$ & 500 & 1000 & 5 & $d 6$ & 1000 & 2000 & 5 & $e 6$ & 2500 & 5000 & 5 \\
$b 5$ & 50 & 100 & 13 & $c 7$ & 500 & 1000 & 10 & $d 7$ & 1000 & 2000 & 10 & $e 7$ & 2500 & 5000 & 10 \\
$b 6$ & 50 & 100 & 25 & $c 8$ & 500 & 1000 & 83 & $d 8$ & 1000 & 2000 & 167 & $e 8$ & 2500 & 5000 & 417 \\
$b 7$ & 75 & 94 & 13 & $c 9$ & 500 & 1000 & 125 & $d 9$ & 1000 & 2000 & 250 & $e 9$ & 2500 & 5000 & 625 \\
$b 8$ & 75 & 94 & 19 & $c 10$ & 500 & 1000 & 250 & $d 10$ & 1000 & 2000 & 500 & $e 10$ & 2500 & 5000 & 1250 \\
$b 9$ & 75 & 94 & 38 & $c 11$ & 500 & 2500 & 5 & $d 11$ & 1000 & 5000 & 5 & $e 11$ & 2500 & 12500 & 5 \\
$b 10$ & 75 & 150 & 13 & $c 12$ & 500 & 2500 & 10 & $d 12$ & 1000 & 5000 & 10 & $e 12$ & 2500 & 12500 & 10 \\
$b 11$ & 75 & 150 & 19 & $c 13$ & 500 & 2500 & 83 & $d 13$ & 1000 & 5000 & 167 & $e 13$ & 2500 & 12500 & 417 \\
$b 12$ & 75 & 150 & 38 & $c 14$ & 500 & 2500 & 125 & $d 14$ & 1000 & 5000 & 250 & $e 14$ & 2500 & 12500 & 625 \\
$b 13$ & 100 & 125 & 17 & $c 15$ & 500 & 2500 & 250 & $d 15$ & 1000 & 5000 & 500 & $e 15$ & 2500 & 12500 & 1250 \\
$b 14$ & 100 & 125 & 25 & $c 16$ & 500 & 12500 & 5 & $d 16$ & 1000 & 25000 & 5 & $e 16$ & 2500 & 62500 & 5 \\
$b 15$ & 100 & 125 & 50 & $c 17$ & 500 & 12500 & 10 & $d 17$ & 1000 & 25000 & 10 & $e 17$ & 2500 & 62500 & 10 \\
$b 16$ & 100 & 200 & 17 & $c 18$ & 500 & 12500 & 83 & $d 18$ & 1000 & 25000 & 167 & $e 18$ & 2500 & 62500 & 417 \\
$b 17$ & 100 & 200 & 25 & $c 19$ & 500 & 12500 & 125 & $d 19$ & 1000 & 25000 & 250 & $e 19$ & 2500 & 62500 & 625 \\
$b 18$ & 100 & 200 & 50 & $c 20$ & 500 & 12500 & 250 & $d 20$ & 1000 & 25000 & 500 & $e 20$ & 2500 & 62500 & 1250 \\
$c 1$ & 500 & 625 & 5 & $d 1$ & 1000 & 1250 & 5 & $e 1$ & 2500 & 3125 & 5 & & & \\
$c 2$ & 500 & 625 & 10 & $d 2$ & 1000 & 1250 & 10 & $e 2$ & 2500 & 3125 & 10 & &
\end{tabular}

Tables 2-4 show the computational results of models $\left(\mathrm{STP}_{3}\right)$ and $\left(\mathrm{LSTP}_{3}\right)$ for the STP OR-Library instances of type $b, c, d$ and $e$. Of the 78 instances available in the Library, 49 were successfully executed, and the others required an amount of computational memory above the capacity of the available computer.

Table 2 shows that the linear relaxation $\left(\mathrm{LSTP}_{3}\right)$ has performed faster than the integer model $\left(\mathrm{STP}_{3}\right)$ in $55.56 \%$ of type $b$ instances. Besides, both models were able to find all the optimal integer solutions for these instances.

Table 3 shows that the linear relaxation $\left(\mathrm{LSTP}_{3}\right)$ has performed faster than the integer model $\left(\mathrm{STP}_{3}\right)$ in $63.16 \%$ of type $b$ instances. In addition, both models were able to find all the optimal integer solutions for these instances, except for instance $c 18$ where $\operatorname{LSTP}_{3}$ found 11.21 instead of 113 .

Table 4 shows that the linear relaxation $\left(\mathrm{LSTP}_{3}\right)$ has performed faster than the integer model $\left(\mathrm{STP}_{3}\right)$ for all the instances of types $d$ and $e$ that could be solved to optimality, except for instance $e 7$ that could only be solved by the integer model. 
TABLE 2. Computational results of $\left(\mathrm{STP}_{3}\right)$ and $\left(\mathrm{LSTP}_{3}\right)$ for STP OR-Library instances $b$.

\begin{tabular}{crll}
\hline \hline Instance & Optimal solution & $\operatorname{Time~STP}_{3}(\mathrm{~s})$ & $\operatorname{Time~LSTP}_{3}(\mathrm{~s})$ \\
\hline$b 1$ & 82 & 0.03 & 0.01 \\
$b 2$ & 83 & 0.02 & 0.02 \\
$b 3$ & 138 & 0.03 & 0.03 \\
$b 4$ & 59 & 0.03 & 0.03 \\
$b 5$ & 61 & 0.03 & 0.03 \\
$b 6$ & 122 & 0.12 & 0.12 \\
$b 7$ & 111 & 0.04 & 0.03 \\
$b 8$ & 104 & 0.03 & 0.04 \\
$b 9$ & 220 & 0.09 & 0.09 \\
$b 10$ & 86 & 0.09 & 0.06 \\
$b 11$ & 88 & 0.10 & 0.10 \\
$b 12$ & 174 & 0.48 & 0.45 \\
$b 13$ & 165 & 0.45 & 0.44 \\
$b 14$ & 235 & 0.11 & 0.11 \\
$b 15$ & 318 & 0.15 & 0.15 \\
$b 16$ & 127 & 0.13 & 0.13 \\
$b 17$ & 131 & 0.20 & 0.20 \\
$b 18$ & 218 & 0.60 & 0.60 \\
\hline
\end{tabular}

TABLE 3. Computational results of $\left(\mathrm{STP}_{3}\right)$ and $\left(\mathrm{LSTP}_{3}\right)$ for STP OR-Library instances $c$.

\begin{tabular}{cccc}
\hline \hline Instance & Optimal solution & ${\text { Time } \mathrm{STP}_{3}(\mathrm{~s})}$ & Time $\mathrm{LSTP}_{3}(\mathrm{~s})$ \\
\hline 1 & 85 & 0.14 & 0.06 \\
$c 2$ & 144 & 0.49 & 0.29 \\
$c 3$ & 754 & 5.66 & 6.80 \\
$c 4$ & 1079 & 23.60 & 22.98 \\
$c 5$ & 1579 & 60.81 & 71.32 \\
$c 6$ & 55 & 0.69 & 0.18 \\
$c 7$ & 102 & 1.49 & 1.44 \\
$c 8$ & 509 & 149.86 & 205.77 \\
$c 9$ & 707 & 1004.31 & 2218.25 \\
$c 10$ & 1093 & 1865.51 & 575.52 \\
$c 11$ & 32 & 2.85 & 1.52 \\
$c 12$ & 46 & 15.91 & 7.62 \\
$c 13$ & 258 & 4130.90 & 10246.80 \\
$c 14$ & 323 & 7076.49 & 119248.00 \\
$c 15$ & 556 & 12.74 & 5.94 \\
$c 16$ & 11 & 57.15 & 39.41 \\
$c 17$ & 18 & 48009.80 & 31724.10 \\
$c 18$ & $113\left(\mathrm{LSTP}_{3}=112.21\right)$ & 361601.00 & 110588.00 \\
$c 19$ & 146 & & \\
\hline
\end{tabular}

In all but one Beasley problems for which we had enough memory to solve through the linear relaxation $\left(\mathrm{LSTP}_{3}\right)$ we were able to find an integer solution. Instance $c 18$ was the only exception. However, as the value of its objective function was equal to 112.21 and all coefficients are integer, the bound to be considered is indeed 113 . 
TABLE 4. Computational results of $\left(\mathrm{STP}_{3}\right)$ and $\left(\mathrm{LSTP}_{3}\right)$ for STP OR-Library instances $d$ and $e$.

\begin{tabular}{cccc}
\hline \hline Instance & Optimal solution & $\operatorname{Time~}^{2} \mathrm{STP}_{3}(\mathrm{~s})$ & $\operatorname{Time~LSTP}_{3}(\mathrm{~s})$ \\
\hline$d 1$ & 106 & 1.37 & 0.29 \\
$d 2$ & 220 & 1.92 & 0.34 \\
$d 6$ & 67 & 6.13 & 1.27 \\
$d 7$ & 103 & 11.75 & 2.78 \\
$d 11$ & 29 & 8.13 & 5.76 \\
$d 12$ & 42 & 49.80 & 37.59 \\
$d 16$ & 13 & 30.60 & 22.17 \\
$e 1$ & 11 & 1.06 & 0.55 \\
$e 2$ & 214 & 3.64 & 1.77 \\
$e 6$ & 73 & 10.10 & 4.08 \\
$e 7$ & 145 & 84.64 & Not enough memory \\
\hline
\end{tabular}

\section{Conclusions And suggestions For Future WORK}

The hypothesis initially formulated was that linear relaxations derived from multiflow formulations would be good (strong) approximations for the Steiner problem in graphs.

The computational results presented for the STP OR-Library (J.E. Beasley) instances of type $b, c, d$ and $e$ corroborated the initial hypothesis, since the linear relaxation $\mathrm{LSTP}_{3}$ presented, in a faster way, the same results obtained by the integer model.

In addition, it is worth mentioning that the multiflow mixed integer linear formulations presented have a polynomial number (although very large) of variables and constraints.

As future work we suggest two fronts. The first one is related to the application of VUB (variable upper bound) techniques [12] to constraints (3.10) in model $\mathrm{STP}_{3}$, as did in [10]. We think this can lead us to solve much larger problems.

The second front consists in using more sophisticated algorithms, such as Lagrangian relaxation algorithms, for the resolution of formulation $\mathrm{STP}_{3}$. The works $[1,2]$ have already shown that the Volume Algorithm and the Revised Volume Algorithm work very well for these kind of multiflow formulations. Besides, the suboptimal primal solutions generated by these algorithms can be used as a starting point for a Simplex-based method or for the development of heuristics and metaheuristics that generate better primal solutions.

Acknowledgements. This work was partially supported by the National Council for Scientific and Technological Development - CNPq, under Grants 305180/2016-9 and 302578/2014-5. The authors also thank CAPES-MEC and COPPETEC.

\section{REFERENCES}

[1] L. Bahiense, N. Maculan and C. Sagastizábal, The volume algorithm revisited: relation with bundle methods. Math. Program. 94 (2002) 41-69.

[2] L. Bahiense, F. Barahona and O. Porto, Solving Steiner tree problems in graphs with lagrangian relaxation. J. Comb. Optim. 7 (2003) 259-282.

[3] J.E. Beasley, An algorithm for the Steiner problem in graphs. Networks 14 (1984) 147-159.

[4] A. Besso, Problema de Steiner em grafos: Uma experiência numérica para problemas de médio porte utilizando formulações compactas de multi-fluxo. Master's thesis. Programa de Engenharia de Sistemas e Computação, COPPE, Federal University of Rio de Janeiro, Rio de Janeiro (2015).

[5] A. Claus and N. Maculan, Une nouvelle formulation du problème de Steiner sur un graphe orienté. In: Publication 315, Centre de Recherche sur les Transports, Université de Montréal, Montréal (1983).

[6] M.X. Goemans and Y.S. Myung, A catalog of Steiner tree formulations. Networks 23 (1993) 19-28.

[7] F.K. Hwang, D.S. Richards and P. Winter, The Steiner tree problem. In: Vol. 53 of Annals of Discrete Mathematics. North-Holland, Amsterdam (1992). 
[8] R.M. Karp, Reducibility among combinatorial problems, edited by R.E. Miller, J.W. Thatcher and J.D. Bohlinger. In: Complexity of Computer Computations. The IBM Research Symposia Series. Springer, New York, NY (1972) 85-103.

[9] N. Maculan, The Steiner problem in graphs. Ann. Discrete Math. 31 (1987) 185-211.

[10] N. Maculan, D. Arpin and S. Nguyen, Le problème de Steiner sur un graphe orienté: formulations et relaxations. Comput. Appl. Math. 7 (1988) 109-118.

[11] N. Maculan, G. Plateau and A. Lisser, Integer linear models with a polynomial number of variables and constraints for some classical combinatorial optimization problems. Pesquisa Oper. 23 (2003) 161-168.

[12] L. Schrage, Implicit representation of generalized variable upper bounds in linear programming. Math. Program. 14 (1978) $11-20$.

[13] R.T. Wong, A dual ascent approach to Steiner tree problems on a directed graph. Math. Program. 28 (1984) $271-287$. 